\title{
ANÁLISE DA VARIABILIDADE PLUVIAL NA UNIDADE DE GERENCIAMENTO DE RECURSOS HÍDRICOS DO PARAÍBA DO SUL (UGHRI-2)
}

\author{
MORAES, Mariana de Paula Costa - prof.marianamoraes@gmail.com \\ Mestranda em Geografia pelo Instituto de Geociências da Universidade Estadual de Campinas - UNICAMP
}

NERY, Jonas Teixeira - jonas@ourinhos.unesp.br

Prof. Livre Docente da Universidade Estadual Paulista - UNESP Campus de Ourinhos

\begin{abstract}
RESUMO: O estudo de variabilidade se torna cada vez mais importante nos dias atuais. Estudar o comportamento da precipitação perante eventos externos é de suma importância. A região do Vale do Paraíba é importante para o estudo da variabilidade, uma vez que, a região sofre influência da maritimidade e de constantes frentes frias, que acabam ocasionando precipitações durante a maior parte dos meses do ano. Este estudo tem como objetivo analisar a variabilidade pluvial na UGRHI-2, analisando a interferência dos eventos ENOS/Oscilação Sul e da Zona de Convergência do Atlântico Sul (ZCAS) na quantidade e na distribuição de chuvas na região. As UGRHI foram criadas para ajudara distribuição e o controle das águas no estado de São Paulo, dividiu-se as bacias hidrográficas para que assim fossem evitados problemas como os de má distribuição e escassez de água em algumas áreas do estado. Para o estudo da variabilidade, foram utilizados diversos softwares, dentre eles o Excel, Variowin e o pacote estatístico R, na subrotina Climatol, com o objetivo de elaborar isolinhas que mostrem a espacialização das anomalias de chuva nos anos estudados Também foi estudado o Índice de Anomalia de Chuva (IAC), constatando de maneira mais efetiva os anos de anomalia positiva e negativa, com o propósito de estudar a variabilidade temporal da chuva na área de estudo.
\end{abstract}

Palavras-chave: Variabilidade pluvial, UGRHI-2, Vale do Paraíba, isolinhas, IAC VARIABILITY ANALYSIS OF RAIN IN UNIT MANAGEMENT OF WATER RESOURCES OF SOUTH PARAÍBA (UGHRI-2)

ABSTRACT: The study of variability becomes increasingly important nowadays. Studying the behavior of rainfall before external events is of paramount importance. The region of Vale do Paraíba, it is important to study variability, since the region is influenced by the ocean and constant cold fronts that end causing precipitation during most months of the year. This study aims to analyze the variability in rain UGRHI - 2 by analyzing the interference of ENSO events / Southern Oscillation and the Convergence Zone South Atlantic (SACZ) in the amount and distribution of rainfall. The UGRHI helped were created for distribution and control of water in the state of São Paulo, divided watersheds were avoided so that problems such as poor distribution and water shortages in some areas of the state. To study variability, various software, including Excel , Variowin and R statistical package, the subroutine Climatol were used , with the goal of developing isolines showing the spatial distribution of rainfall anomalies in the years studied also the anomaly index was studied rain (IAC), noting more effectively the years of positive and negative anomaly, with the purpose of studying the temporal variability of rainfall in the study area .

\section{1- INTRODUÇÃO}

A UGRHI-2 compreende o total de 34 municípios inseridos no Vale do Paraíba. Localiza-se na porção sudeste do estado de São Paulo. Seus limites são: ao norte, com a UGRHI-1 (Serra da Mantiqueira), além do estado de Minas Gerais; a nordeste, com a UGRHI-5 (Piracicaba/Capivari/Jundiaí); a oeste, novamente com a UGRHI-5 e com a UGRHI-6 (Alto Tietê), sendo que esta última também faz o limite sudoeste; ao sul, com a UGRHI-3 (litoral Norte); a sudeste, também com a UGRHI-3 e com o Estado do Rio de Janeiro e a leste e nordeste, com os Estados do Rio de Janeiro e Minas Gerais, (PLANO ESTADUAL DE RECURSOS HÍDRICOS, 1990). A Figura 1 mostra a localização da área de estudo. 


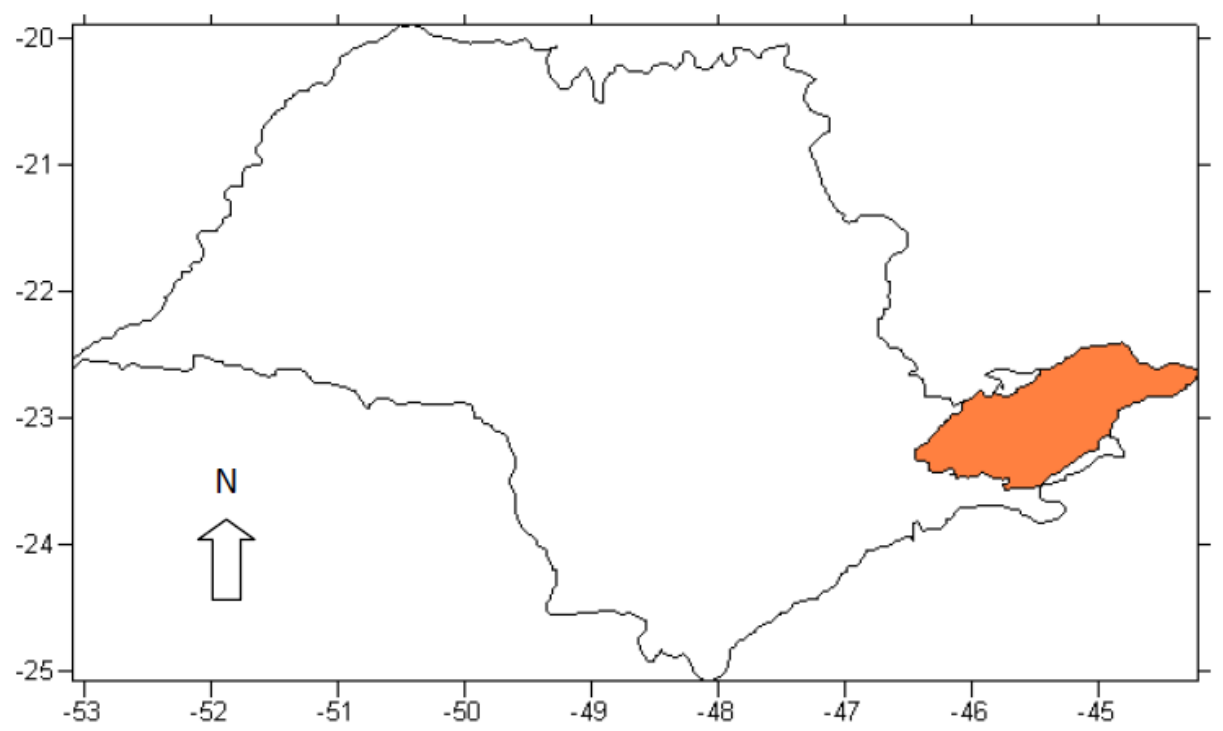

Figura 1 - Localização da UGRHI-2.

De acordo com Marengo e Alves (2005), o clima da bacia hidrográfica do Paraíba do Sul é caracterizado como

Subtropical quente, com temperatura média anual oscilando entre $18^{\circ} \mathrm{C}$ e $24^{\circ} \mathrm{C}$. As máximas precipitações ocorrem nas cabeceiras mineiras da bacia e nos pontos mais altos das serras do Mar e Mantiqueira, chegando a valores de $2.250 \mathrm{~mm} / \mathrm{ano}$, (MARENGO E ALVES, p.217, 2005)

O verão é considerado o período chuvoso, apresentando nos meses de máxima precipitação, acumulado entre 200 e $250 \mathrm{~mm} / \mathrm{mês}$. Já o inverno é o período seco, sendo o intervalo entre os meses de maio e agosto, o mais seco, com precipitação acumulada de $50 \mathrm{~mm} / \mathrm{mês,}$ (MARENGO E ALVES, 2005).

Conti (1973) afirma que a região é constantemente influenciada por sistemas frontais, o que ocasiona precipitações, " mesmo considerando que as chuvas frontais diminuem com a latitude, essa é uma faixa onde os eventos pluviais são determinados predominantemente ela ação das frentes", (CONTI, 1973).

Estudos de variabilidade pluvial se tornam importantes em regiões como o Vale do Paraíba, onde desastres naturais ocasionados por eventos extremos de precipitação ocorrem.

É necessário estudar o comportamento da precipitação e quais são os sistemas, elementos e fatores que influenciam em sua localidade e quantidade.

SANSIGOLO et al. (2004), afirma que em vários estudos sobre variabilidade pluvial, a associação entre oceano e atmosfera tem sido cada vez mais estudada. Esses estudos têm mostrado que os padrões da temperatura da superfície do mar nos oceanos Pacífico e Atlântico podem ser importantes para definir a precipitação em algumas regiões do Brasil, como norte, sul e sudeste.

Não se sabe as reais causas do fenômeno ENOS, mas aparentemente, são internas ao oceano - aparentemente a diferença de pressão ao nível do mar entre o Tahiti e Darwin, no Oceano Pacífico Tropical, está relacionado com o aquecimento anômalo de águas frequentemente frias do lado leste do oceano. Possui duas fases:

- Fase Quente (El Niño): ocorrem anomalias positivas, ou seja, há um aquecimento da Temperatura da superfície do mar (TSM) do Pacífico Equatorial Oriental e Central. Os dois 
principais eventos de El Niño do século aconteceram em 1982/83 e 1997/98, (DESSAY, et al., 2002)

- Fase Fria (La Niña): MARENGO E SAMPAIO (s/d), afirmam que a La Niña ou a fase fria do Oceano Pacífico, é o resfriamento anômalo das águas superficiais do Pacífico Equatorial Central e Oriental.

De acordo com Ferreira, et al. (2004), a Zona de Convergência do Atlântico Sul (ZCAS) é um fenômeno que ocorre no verão da América da Sul. Quadro (1994) afirma que:

Durante os meses de verão sobre a América do Sul grande parte da precipitação é ocasionada pela intensa atividade convectiva sobre o continente. Simultaneamente, a presença das ZCAS, devido a sua estacionaridade, contribui intensamente para a persistência da precipitação em algumas regiões do Brasil. Acredita-se que as ZCAS seja a resposta para a associação de vários sistemas atmosféricos que compõem a circulação de verão sobre a América do Sul, (QUADRO, p.7, 1994).

Este trabalho tem como principal objetivo relacionar a variabilidade pluvial da área com o evento ENOS- Oscilação Sul e a presença das ZCAS.

\section{2 - MATERIAIS E MÉTODOS}

Para o estudo foram recolhidos dados da hidroweb, site da Agência Nacional de Águas (ANA). Foram utilizadas 14 estações espalhadas pela UGRHI-2, buscando as melhores séries temporais e espaciais (Figura 2). O período escolhido foi 1960 a 2010. A série foi homogeneizada (agrupamento de três) através de um "pacote" estatístico R (software livre) e sua sub-rotina Climatol:

"The "Climatol" $R$ contributed package is mostly devoted to the problem of homogenizing climatological series, that is to say, remove the perturbations produced by changes in the conditions of observation or in the nearby environment to allow the series to reflect only (as far as possible) the climatic variations" (GUIJARRO, p.2, 2011).

\begin{tabular}{|c|c|c|c|c|}
\hline Número & Nome da estação & Cidade - SP & Longitude & Latitude \\
\hline 1 & $\begin{array}{l}\text { Queluz (Centro } \\
\text { Saúde) }\end{array}$ & $\overline{\text { Queluz }}$ & $-44,77$ & $-22,53$ \\
\hline 2 & $\begin{array}{l}\text { Fazenda Santa } \\
\text { Clara }\end{array}$ & $\begin{array}{l}\text { Cachoeira } \\
\text { Paulista }\end{array}$ & $-44,97$ & $-22,68$ \\
\hline 3 & Campos de Cunha & Cunha & $-44,82$ & $-22,92$ \\
\hline 4 & Bananal & Bananal & $-44,32$ & $-22,68$ \\
\hline 5 & Guaratinguetá & Guaratinguetá & $-45,17$ & $-22,80$ \\
\hline 6 & Pindamonhangaba & Pindamonhangaba & $-45,47$ & $-22,90$ \\
\hline 7 & Estrada de Cunha & Cunha & $-45,03$ & $-22,98$ \\
\hline 8 & Taubaté & Taubaté & $-45,55$ & $-23,03$ \\
\hline 9 & Caçapava & Caçapava & $-45,70$ & $-23,07$ \\
\hline 10 & $\begin{array}{l}\text { São Luís do } \\
\text { Paraitinga }\end{array}$ & $\begin{array}{l}\text { São Luís do } \\
\text { Paraitinga }\end{array}$ & $-45,32$ & $-23,22$ \\
\hline 11 & Santa Branca & Santa Branca & $-45,90$ & $-23,37$ \\
\hline 12 & Usina Bocaina & $\begin{array}{l}\text { Cachoeira } \\
\text { Paulista }\end{array}$ & $-44,90$ & $-22,72$ \\
\hline 13 & $\begin{array}{l}\text { São José do } \\
\text { Barreiro }\end{array}$ & $\begin{array}{l}\text { São José do } \\
\text { Barreiro }\end{array}$ & $-44,57$ & $-22,63$ \\
\hline 14 & Ponte Alta 1 & $\begin{array}{l}\text { São Luís do } \\
\text { Paraitinga }\end{array}$ & $-45,14$ & $-23,33$ \\
\hline
\end{tabular}

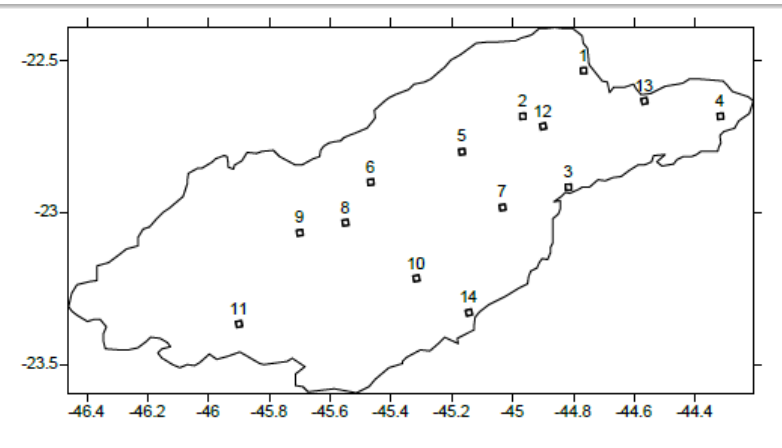

Figura 2 - Estações utilizadas no estudo 
Foram realizados cálculos para as três séries: média climatológica, precipitação anual, desvio padrão e coeficiente de variação.

Todos os dados foram organizados e calculados no software Excel. Os resultados desses cálculos foram colocados espacialmente através de isolinhas, traçadas no software Surfer e para que as isolinhas ficassem mais próximas do real, a Geoestatística foi um recurso utilizado através do software Variowin.

O Índice de Anomalia de Chuva (IAC) analisa a frequência que ocorrem anos secos e chuvosos e a intensidade do evento. Foi desenvolvido por Rooy (1965). Logo, sua utilização em estudos de variabilidade torna-se imprescindível. Tem como fórmula:

$$
\begin{gathered}
I A C=3\left\lfloor\frac{(p-\bar{p})}{(m-\bar{p})}\right\rfloor \text {,para anomalias positivas (1) } \\
I A C=-3\left\lfloor\frac{(p-\bar{p})}{(m-\bar{p})}\right\rfloor \text {, para anomalias negativas (2) }
\end{gathered}
$$

Onde: $p=$ precipitação ou anual $(\mathrm{mm}) ;=$ precipitação média anual da série histórica $(\mathrm{mm}) ; m$ $=$ média das dez maiores precipitações anuais da série histórica $(\mathrm{mm}) ; x=$ média das dez menores precipitações anuais da série histórica $(\mathrm{mm})$; e anomalias positivas são valores acima da média, e negativas, abaixo da média.

A Tabela 1 mostra a classificação do IAC:

\begin{tabular}{cc}
\hline IAC & Classificação \\
\hline$\geq 4,00$ & Extremamente úmido \\
\hline 3,00 a 3,99 & Umidade alta \\
2,00 a 2,99 & Umidade moderada \\
0,5 a 1,99 & Umidade baixa \\
$-0,49$ a 0,49 & Normal \\
$-1,99$ a $-0,5$ & Seca suave \\
$-2,00$ a $-2,99$ & Seca moderada \\
$-3,00$ a $-3,99$ & Seca alta \\
$\leq-4,00$ & Extremamente seca \\
\hline Fonte: EMBRAPA (2009), adaptado pela autora (2010) \\
Tabela 1 - Classificação do IAC
\end{tabular}

Para a análise dos anos de evento ENOS, foi utilizado como base, as tabelas do CPTEC- INPE, que mostram os anos do evento (Tabela 2 e 3 ): 


\begin{tabular}{ll}
\hline $1877-1878$ & $1888-1889$ \\
\hline $1896-1897$ & 1899 \\
$1902-1903$ & $1905-1906$ \\
$1911-1912$ & $1913-1914$ \\
$1918-1919$ & 1923 \\
$1925-1926$ & 1932 \\
$1939-1941$ & $1946-1947$ \\
1951 & 1953 \\
$1957-1959$ & 1963 \\
$1965-1966$ & $1968-1970$ \\
$1972-1973$ & $1976-1977$ \\
$1977-1978$ & $1979-1980$ \\
$1982-1983$ & $1986-1988$ \\
$1990-1993$ & $1994-1995$ \\
$1997-1998$ & $2002-2003$ \\
$2004-2005$ & $2006-2007$ \\
$2009-2010$ & - \\
\hline
\end{tabular}

Tabela 2 - Ocorrências de eventos El Niño

\begin{tabular}{ll}
\hline 1886 & $1903-1904$ \\
\hline $1906-1908$ & $1909-1910$ \\
$1916-1918$ & $1924-1925$ \\
$1928-1929$ & $1938-1939$ \\
$1949-1951$ & $1954-1956$ \\
$1964-1965$ & $1970-1971$ \\
$1973-1976$ & $1983-1984$ \\
$1984-1985$ & $1988-1989$ \\
$1995-1996$ & $1998-2001$ \\
$2007-2008$ & - \\
\hline
\end{tabular}

Fonte: CPTEC/INPE - http://enos.cptec.inpe.br/

Tabela 3 - Ocorrências de eventos La Niña

\section{3 - RESULTADOS E DISCUSSÃO}

As anomalias foram calculadas para os anos de El Niño forte ou muito forte: 1982, 1983, 2009 e 2010.

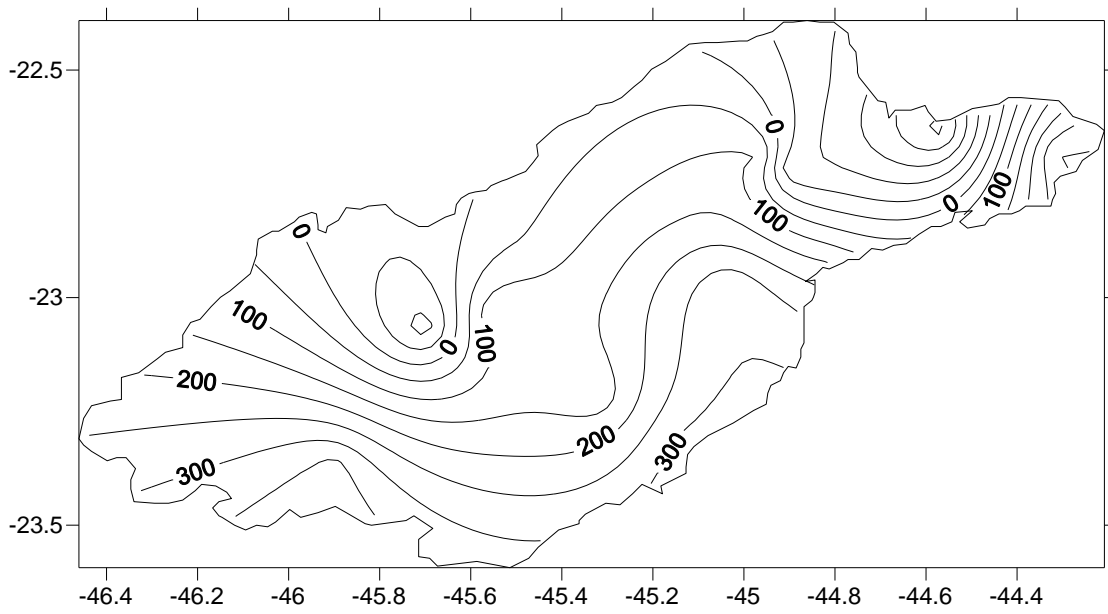

Figura 3 - Anomalias de 1982 


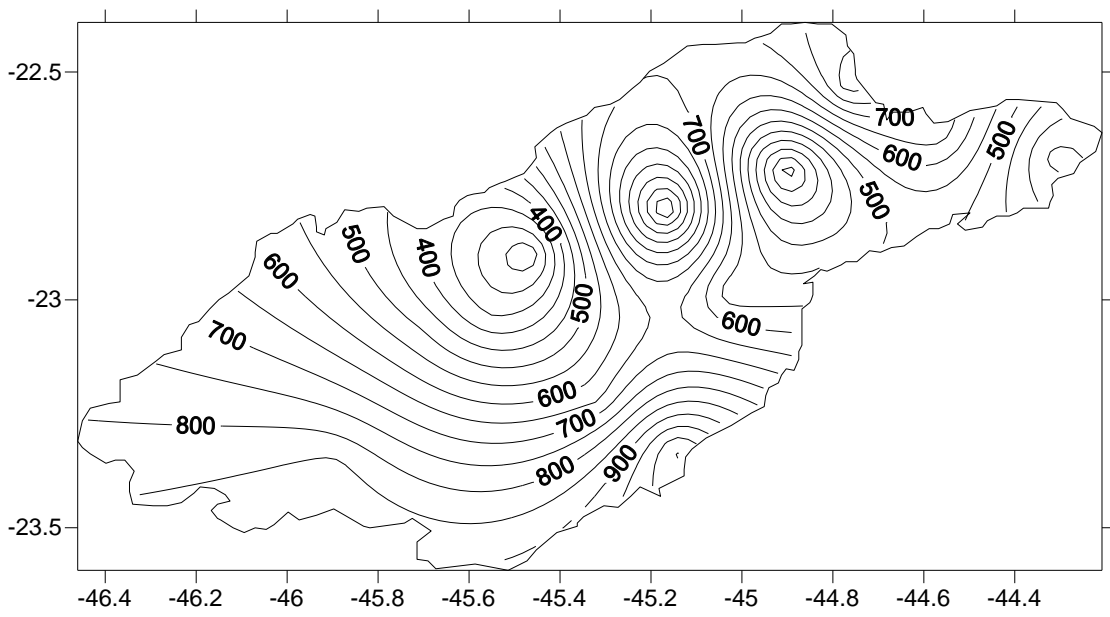

Figura 4 - Anomalias de 1983

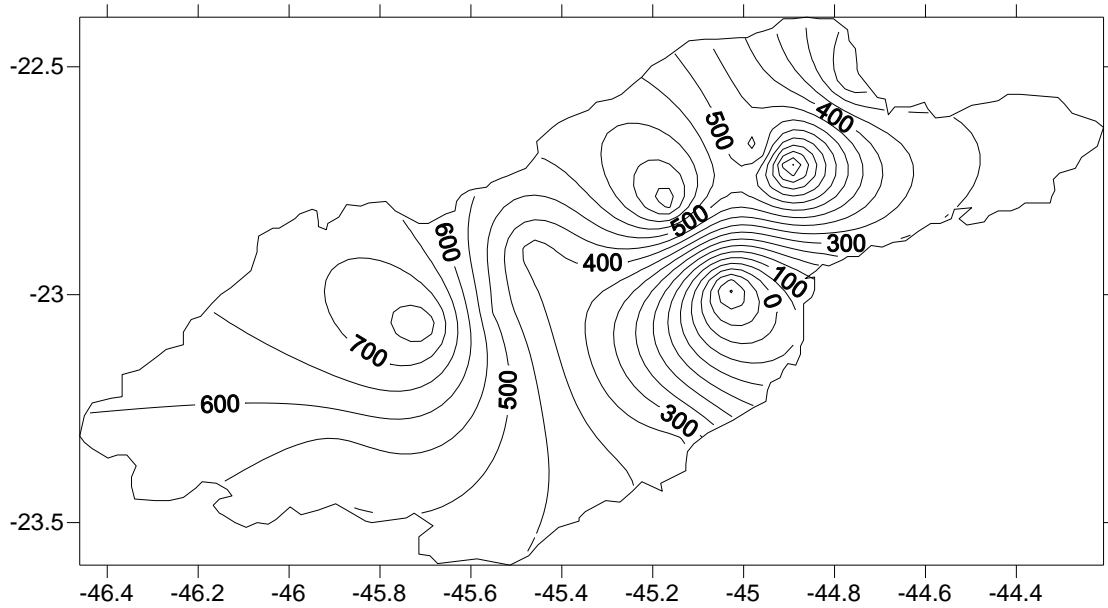

Figura 5 - Anomalias de 2009

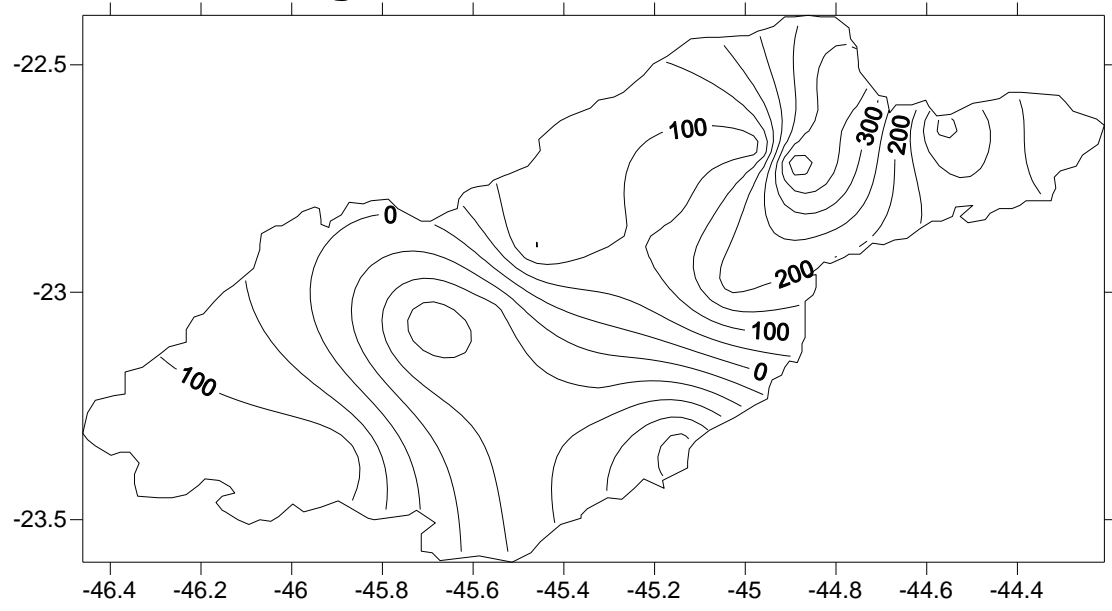

Figura 6 - Anomalias de 2010

Nos anos de El Niño estudados, observa-se que 1983 (Figura 4), foi o ano que mais de destacou, apresentando as maiores anomalias. Essas anomalias estão bem distribuídas pela região, onde os pontos mais altos da bacia apresentam os maiores valores de anomalias.

O ano 2010 apresentou as menores anomalias em relação aos demais, atingindo áreas com valor zero, ou seja, nota-se que o El Niño de 1982-83 foi de maior intensidade que o de 200910 , as anomalias foram maiores e bem mais distribuídas pela região, que apresentou valores altos em todas as suas áreas.

As Figuras 7 a 10 apresentam os anos de La Niña. 


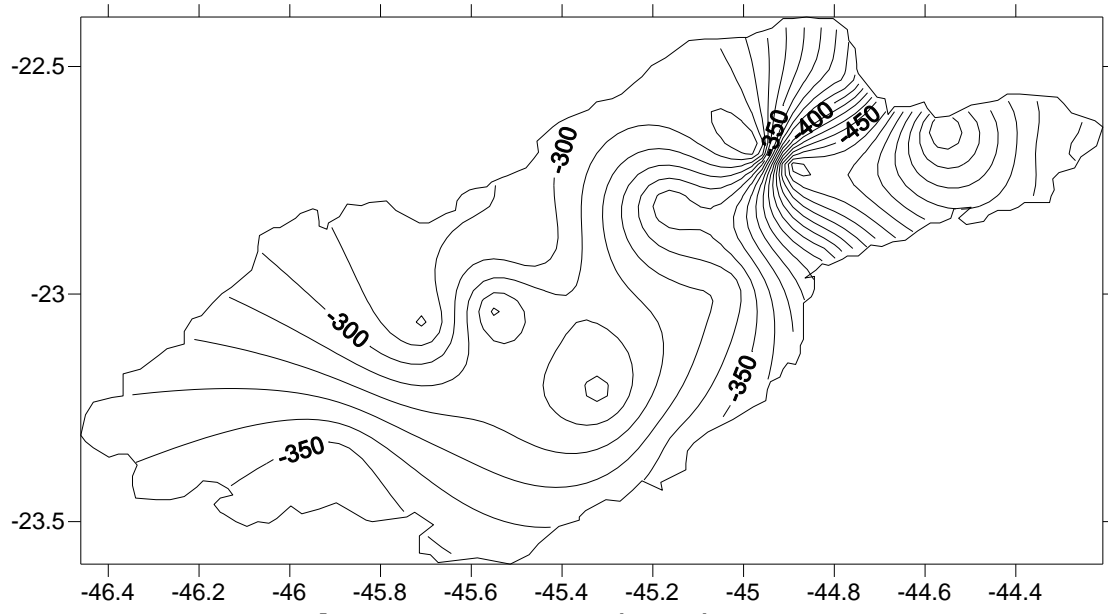

Figura 7 - Anomalias de 1984

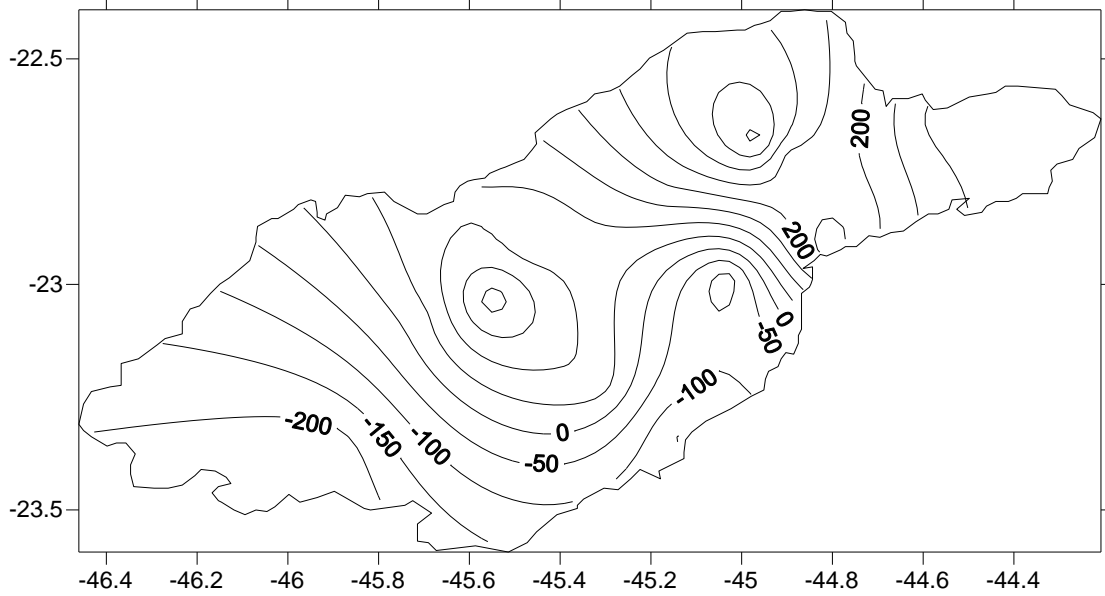

Figura 8 - Anomalias de 1985

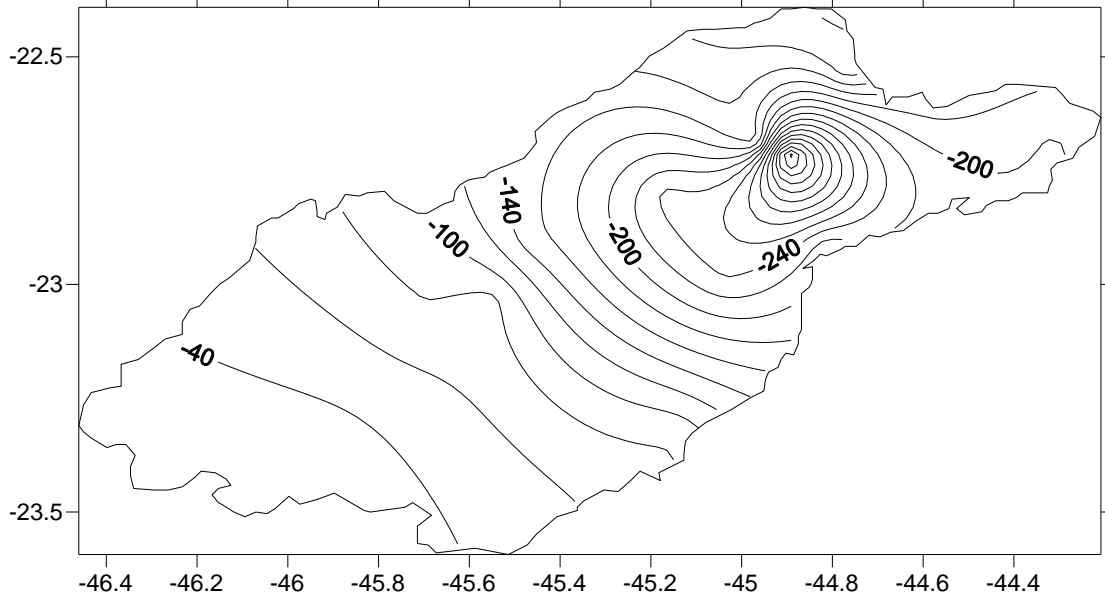

Figura 9 - Anomalias de 2007 


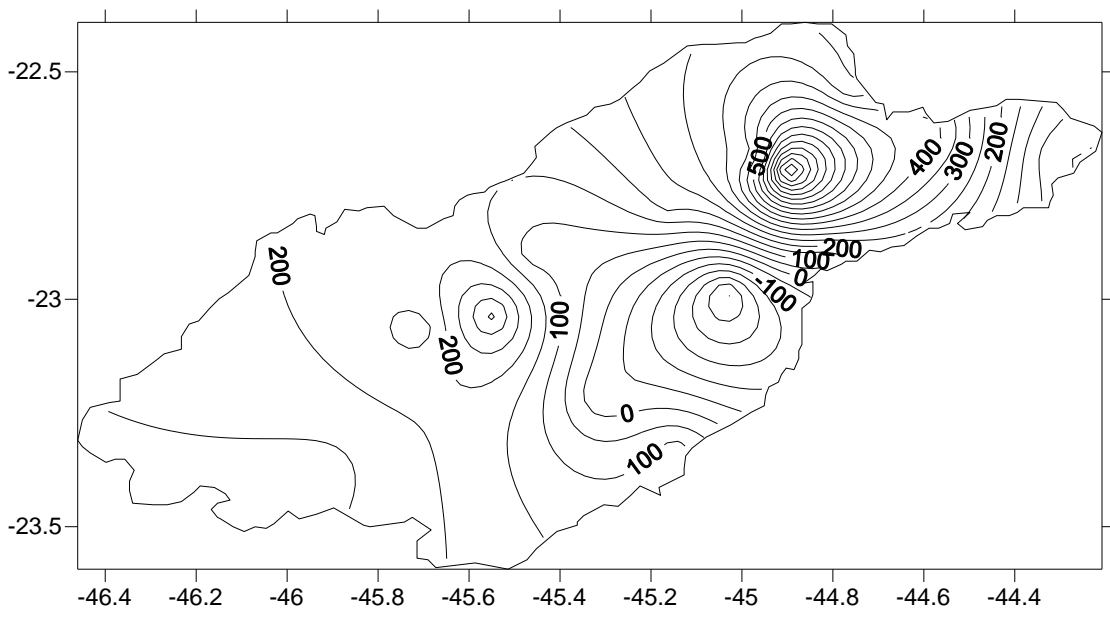

Figura 10 - Anomalias de 2008

É possível observar (Figuras 7 a 10) que 1984 foi o ano que teve La Niña mais marcante, seguido de 1985 e 2007. Observa-se em 1984, que as anomalias negativas chegaram a -500 $\mathrm{mm}$, ou seja, $500 \mathrm{~mm}$ abaixo da média climatológica.

Na Figura 10, pode-se observar que o ano 2008 não apresentou La Niña marcante na região. Uma das respostas para isso pode ser que 2008 tenha sido um ano de transição entre La Niña e El Niño, logo a La Niña já estava perdendo força, não influenciando de maneira tão direita na precipitação da região.

O IAC foi calculado para todas as estações, mas como exemplo, aqui serão mostrados apenas dois gráficos, de duas estações da UGRHI-2.

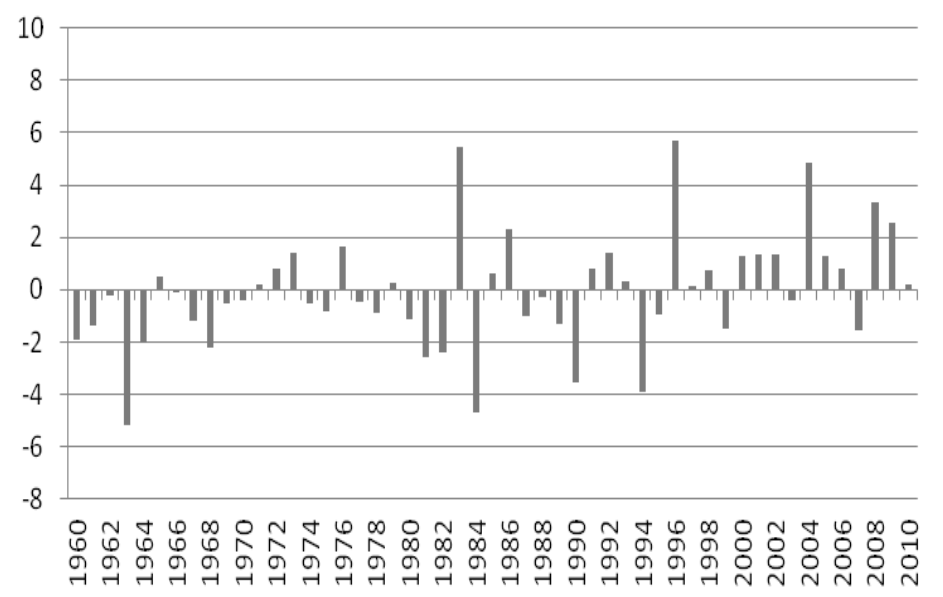

Figura 11 - Distribuição temporal do IAC para a estação São José do Barreiro. 


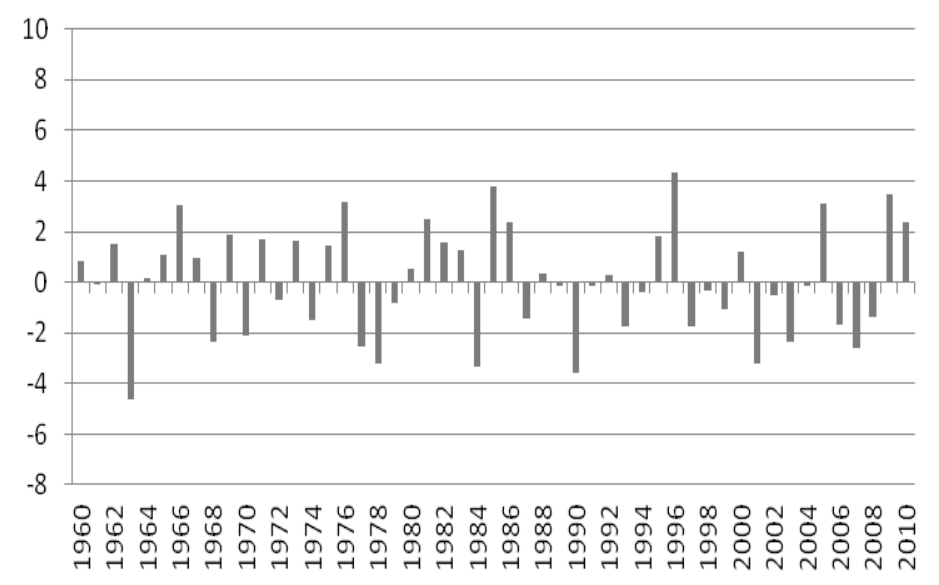

Figura 12 - Distribuição temporal do IAC para a estação Ponta Alta 1.

Nas Figuras 11 a 12, pode-se observar marcada variabilidade temporal, ou seja, os valores de IAC variaram ao passar dos anos, possuindo picos muito altos em alguns anos e picos muito baixos em outros. É possível relacionar esse índice com o ENOS, pois os anos que apresentaram altos valores de IAC são justamente os anos de El Niño, como pode ser observado no anos de 1983 em quase todos os gráficos. Já os anos que possuem valores baixos de IAC, são geralmente anos de La Niña, como pode ser visto no ano 1984.

Abaixo segue a classificação do IAC (Tabela 4). destacando os anos úmidos e secos: 


\begin{tabular}{|c|c|c|c|c|c|c|c|c|c|c|c|c|c|c|}
\hline \multirow[t]{2}{*}{ ANO } & \multicolumn{14}{|c|}{ ESTAÇÕES } \\
\hline & 1 & 2 & 3 & 4 & 5 & 6 & 7 & 8 & 9 & 10 & 11 & 12 & 13 & 14 \\
\hline 1972 & SCS & UMB & UMB & UMB & $\mathrm{SCM}$ & SCS & SCS & NOR & SCS & SCS & SCS & NOR & UMB & SCS \\
\hline 1973 & SCS & UMM & SCS & SCS & SCS & UMB & SCS & NOR & NOR & $\mathrm{SCM}$ & SCS & SCS & UMB & UMB \\
\hline 1982 & SCS & UMB & UMB & UMB & UMB & NOR & UMM & UMB & SCS & UMB & UMM & SCS & $\mathrm{SCM}$ & UMB \\
\hline 1983 & EXU & UMA & EXU & EXU & EXU & UMB & UMA & UMM & EXU & EXU & EXU & UMB & EXU & UMB \\
\hline 1984 & $\mathrm{SCA}$ & $\mathrm{SCM}$ & EXS & EXS & $\mathrm{SCM}$ & $\mathrm{SCM}$ & SCA & $\mathrm{SCM}$ & $\mathrm{SCA}$ & $\mathrm{SCA}$ & $\mathrm{SCM}$ & $\mathrm{SCA}$ & EXS & $\mathrm{SCA}$ \\
\hline 1985 & UMB & UMA & UMB & UMB & UMB & NOR & SCS & UMB & NOR & UMB & $\mathrm{SCS}$ & UMB & UMB & UMA \\
\hline 1995 & UMB & UMB & UMB & UMB & UMB & UMB & SCS & UMB & EXU & UMA & EXU & UMA & $\mathrm{SCS}$ & UMB \\
\hline 1996 & $\mathrm{SCM}$ & $\mathrm{SCM}$ & EXU & EXU & NOR & UMB & UMB & UMA & NOR & UMB & UMM & EXU & EXU & EXU \\
\hline 2006 & $\mathrm{SCS}$ & SCS & EXS & EXS & NOR & UMB & UMB & SCS & UMB & UMB & UMB & SCS & UMB & SCS \\
\hline 2007 & SCS & SCS & SCS & SCS & SCS & SCS & $\mathrm{SCM}$ & SCS & SCS & SCS & NOR & $\mathrm{SCA}$ & $\mathrm{SCS}$ & $\mathrm{SCM}$ \\
\hline 2008 & UMM & UMA & NOR & NOR & UMB & UMB & $\mathrm{SCM}$ & UMM & UMB & NOR & UMB & EXU & UMA & SCS \\
\hline 2009 & UMB & EXU & UMB & UMB & EXU & UMA & SCS & UMA & EXU & UMA & UMA & UMA & UMB & UMA \\
\hline 2010 & UMA & UMB & UMB & UMB & NOR & UMB & UMB & SCS & $\mathrm{SCM}$ & SCS & UMB & UMM & NOR & UMB \\
\hline
\end{tabular}

Tabela 4 - Classificação dos dados de acordo com IAC

$\mathrm{Na}$ Tabela 4, é possível observar que alguns anos. mesmo sendo anos de El Niño não apresentaram índice alto, mostrando que muitas vezes esse evento não tem influência na UGRHI estudada. A mesma conclusão é válida para os anos de La Niña, muitos anos não apresentaram classificação de seca.

O principal objetivo da elaboração do IAC é auxiliar na análise da variabilidade pluvial no período de 51 anos e pode-se observar que essa variabilidade é marcada no período. mesmo que alguns anos não possam ser relacionados com eventos ENOS. Entretanto, é possível notar que o ano de 1983, marcado por um El Niño forte, teve classificação compatível, ou seja, apresentou classificação de umidade alta a extremamente úmido na maior parte das estações.

\section{3 - CONCLUSÃO}

Neste artigo, foi possível observar a existência de uma variabilidade pluvial na região estudada.

Essa varibilidade foi constatada através dos eventos ENOS/Oscilação Sul e da presença das ZCAS, que atuam diretamente no aumento de chuvas da região. Em anos de El Niño, as chuvas tendem a aumentar e em anos de La Niña, as precipitações tendem a serem mais distribuídas e em menor quantidade pela região.

O IAC mostra uma marcada varibailidade temporal na precipitação durante o tempo de análise, demarcando bem os anos que ocorrem o evento ENOS/Oscilação Sul, mostrando mais uma vez a importância deste evento para a região. 


\section{REFERÊNCIAS}

CONTI, J.B Circulação secundária e efeito orográfico na gênese das chuvas na região lesnordeste paulista. São Paulo,1975, Tese (doutorado), Série Teses e Monografias, IGEOGUSP 18

DESSAY,N., et al. Uma análise das diferenças de comportamentos da vegetação na América do Sul durante os dois eventos de El Niño de 1982-1983 e 1997-1998. XII Congresso Brasileiro de Meteorologia. Foz do Iguaçu, PR, 2002. Disponivel em< http://www.cbmet.com/cbm-files/11-2c197ef010063e3181d61999a23971f8.pdf> Acesso em: 2 de novembro de 2009.

FERREIRA, N.J.; SANCHES, M.; DIAS, M.A.F.S. Composição da zona de convergência do Atlântico Sul em períodos de EI Niño e La Nina. Revista Brasileira de Meteorologia, v.19, n.1, 89-98, 2004. Disponível em www.rbmet.org.br/port/revista/revista_dl.php?id_artigo=45\&id...153> Acesso em 17 de agosto de 2011.

GUIJARRO, J.A. User's guide to climatol. Junho, 2011

MARENGO,J.A; ALVES L,M. Tendências hidrológicas da bacia do rio paraíba do sul. Revista Brasileira de Meteorologia, v.20, n.2, 215-226, 2005. Disponível em < http://webcache.googleusercontent.com/search?q=cache:2nHetTZROmIJ:www.rbmet.org.br/p ort/revista/revista_dl.php\%3Fid_artigo\%3D506\%26id_arquivo\%3D132+\&cd=1\&hl=pt-

BR\&ct $=$ clnk\&gl=br $>$ Acesso em: 20/09/2012

MARENGO, J.A ; OLIVEIRA,G.S. Impactos do fenômeno la niña no tempo e clima do brasil: desenvolvimento e intensificação do la niña 1998/99. s/d. Disponível em <http://webcache.googleusercontent.com/search?q=cache:m0jG4qm_qz0J:mtcm15.sid.inpe.br/col/cptec.inpe.br/walmeida/2004/06.30.08.07/doc/Marengo_Impactos\%2520d o\%2520fenomeno.pdf $+\& c d=1 \& \mathrm{hl}=$ pt-BR\&ct=clnk\&gl=br> Acesso em 20/08/2010

QUADRO, M.F.L. Estudo de episódios de zonas de convergência do Atlântico Sul (ZCAS) sobre a América do Sul. Dissertação de Mestrado em Meteorologia (INPE), São José dos Campos, 1994. Disponível em < http://mtc-m15.sid.inpe.br/col/sid.inpe.br/MTCm13\%4080/2005/08.26.14.10/doc/publicacao.pdf> Acesso em 17 de agosto de 2011.

ROOY, M. P. van. A rainfall anomaly index independent of time and space. Notos, Pretoria, v. 14, p. 43-48, 1965.

SÃO PAULO (ESTADO). Plano Estadual de Recursos Hídricos. Disponível em <http://www.sigrh.sp.gov.br/sigrh/basecon/perh90/Perh9009.htm > Acessado em 25/08/2010.

SANSIGOLO, C. A.; PEREIRA, C.S.; SILVA, I. R. Relações entre as precipitações regionais no Sul do Brasil e as temperaturas da superfície dos oceanos Atlântico e Pacífico. Revista Brasileira de Meteorologia, v.19, n.1, 5-11, 2004 